\title{
Alterstice
}

Revue internationale de la recherche interculturelle

International Journal of Intercultural Research

Revista International de la Investigacion Intercultural

\section{Santé mentale et politique du relationnel. Vers de nouvelles configurations}

\section{Lise Demailly}

Volume 4, numéro 2, 2014

Santé mentale et sociétés plurielles

URI : https://id.erudit.org/iderudit/1077425ar

DOI : https://doi.org/10.7202/1077425ar

Aller au sommaire du numéro

Éditeur(s)

Alterstice

ISSN

1923-919X (numérique)

Découvrir la revue

Citer cet article

Demailly, L. (2014). Santé mentale et politique du relationnel. Vers de nouvelles configurations. Alterstice, 4(2), 49-61. https://doi.org/10.7202/1077425ar
Résumé de l'article

À partir d'une série de travaux empiriques menés depuis une dizaine d'années sur la politique et les pratiques de la santé mentale en France, l'auteur présente une synthèse des reconfigurations contemporaines du champ, sous la pression d'injonctions sociétales à la fois fortes et contradictoires. L'article décrit une réorganisation des " clientèles » des différentes institutions concernées qui fait la part belle d'une part à leur solvabilité et, d'autre part, à leur possibilité de résilience sociale. Se dessinent ainsi de nouvelles politiques du relationnel. La politique dominante est organisée autour du registre de l'intervention, est sensible aux préoccupations sécuritaristes, est à la fois libérale et autoritaire et relève d'une conception essentiellement adaptative de l'autonomie. Les mouvements d'usagers constituent l'une des formes de résistance à la psychiatrie contemporaine, en refusant que l'on fasse leur bien sans eux, voire contre eux. D’autres résistances sont formées par les professionnels et les individus attachés à une conception de l'autonomie comme émancipation. 


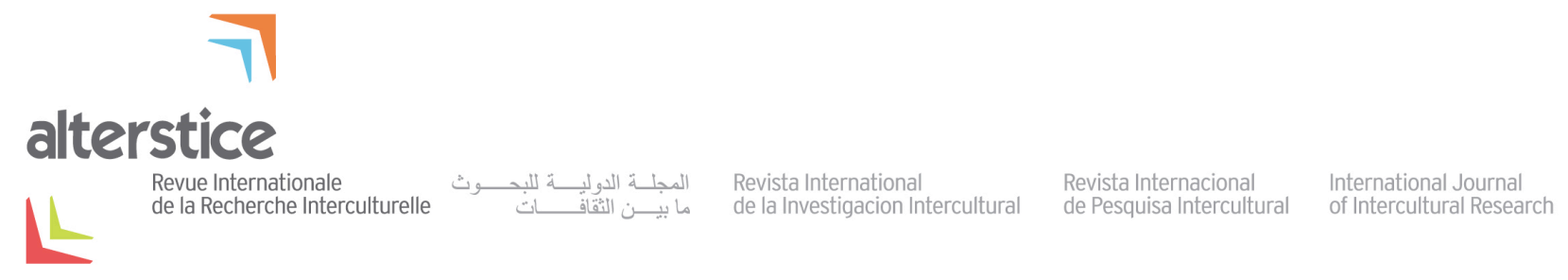

ARTICLE THÉMATIQUE

\section{Santé mentale et politique du relationnel. Vers de nouvelles configurations}

Lise Demailly ${ }^{1}$

\section{Résumé}

À partir d'une série de travaux empiriques menés depuis une dizaine d'années sur la politique et les pratiques de la santé mentale en France, l'auteur présente une synthèse des reconfigurations contemporaines du champ, sous la pression d'injonctions sociétales à la fois fortes et contradictoires. L'article décrit une réorganisation des " clientèles » des différentes institutions concernées qui fait la part belle d'une part à leur solvabilité et, d'autre part, à leur possibilité de résilience sociale. Se dessinent ainsi de nouvelles politiques du relationnel. La politique dominante est organisée autour du registre de l'intervention, est sensible aux préoccupations sécuritaristes, est à la fois libérale et autoritaire et relève d'une conception essentiellement adaptative de l'autonomie. Les mouvements d'usagers constituent l'une des formes de résistance à la psychiatrie contemporaine, en refusant que l'on fasse leur bien sans eux, voire contre eux. D'autres résistances sont formées par les professionnels et les individus attachés à une conception de l'autonomie comme émancipation.

\section{Rattachement de l'auteure}

${ }^{1}$ Université des sciences et techniques de Lille 1 et Centre de recherche et d'études en sociologie et économie (CRESE), unité mixte de recherche (UMR) et Centre national de la recherche scientifique (CNRS), France

\section{Correspondance}

lise.demailly@univ-lille1.fr

\section{Mots clés}

santé mentale; politiques de la relation; psychiatrie

\section{Pour citer cet article}

Demailly, L. (2014). Santé mentale et politique du relationnel. Vers de nouvelles configurations. Alterstice, 4(2), 49-62. 
Ce texte est consacré à l'évolution des pratiques et politiques de santé mentale dans nos sociétés occidentales contemporaines et à leurs enjeux en matière de " politiques de la relation » (Demailly, 2008). II ne s'agit pas de la présentation d'une recherche particulière, mais la reprise réflexive d'un certain nombre d'études empiriques ou théoriques que j'ai menées depuis une dizaine d'années. Ces travaux, pour une partie d'entre eux, ont porté sur l'action publique en santé mentale : observation des modes de coordination entre professionnels du sanitaire et professionnels du social (recherche menée en 2004-2006, voir Demailly et Bresson, 2006), observation du processus de territorialisation de la politique de santé mentale dans le Nord-Pas-de-Calais en France (recherche européenne Knowledge and policy ${ }^{1}$, voir Demailly, Chéronnet et Mossé, 2009), analyse des outils de régulation gestionnaires et statistiques de la psychiatrie française (Autès, Demailly, Dembiski, Bélard et Lardé, 2010), étude du développement de l'évaluation dans les services psychiatriques (Demailly, Devineau, Maury et Mossé, 2010). Ce contrat européen a permis la mise en perspective de la politique française de santé mentale avec celle de l'Écosse, de la Norvège, de la Hongrie et de l'Allemagne.

Une seconde série de travaux empiriques personnels mobilisés dans cet article porte sur des innovations organisationnelles et cliniques en psychiatrie : la mise en place d'un service ouvert en pédopsychiatrie dans un quartier populaire (Demailly et Chéronnet, 2007), des équipes mobiles de psychiatrie (Demailly, Dembinski et Déchamp Le Roux, 2013), la mise en œuvre du programme expérimental (2012-2014) de " médiateurs de santé pairs en santé mentale " (ex-patients, en voie de rétablissement, embauchés dans les services psychiatriques sur contrats de travail à durée déterminée de la fonction publique), qui a donné lieu à un rapport d'évaluation (Demailly, Bélard et collab., 2014).

Enfin plusieurs textes qui précédent celui-ci ont développé des analyses quant aux dynamiques du champ de la santé mentale et notamment des tensions politiques, éthiques et théoriques qui affectent la psychiatrie (Demailly, 2012 et 2014), analyse qui sera poursuivie ici ${ }^{2}$. Les analyses globales des évolutions politiques du champ de la santé mentale ont pu s'appuyer sur des observations de congrès de psychiatrie, de colloques de psychanalyse, de séminaires, de formations continues, de listes de discussions sur internet, de revues et ouvrages professionnels, de prises de parole d'usagers.

Il n'est pas possible d'exposer ici le cadre théorique et la méthodologie de chacun de ces travaux et on renverra le lecteur aux publications mentionnées. Succinctement, la première série de travaux utilise les concepts développés par certains politistes (plus particulièrement Jobert, 1995; Le Gales et Lacousmes, 2005; Palier et Surel, 2005), mais en accordant une place plus importante qu'en sciences politiques à l'enquête par observation in situ sur les pratiques elles-mêmes, selon les méthodes traditionnelles en sociologie du travail, ainsi que sur les effets concrets, dans les mondes vécus ordinaires, des décisions politiques ou juridiques. La seconde série d'études, qui porte sur des innovations organisationnelles et cliniques, a accordé une place centrale à l'observation ethnographique longue des situations d'interaction (réunions formelles ou informelles, entretiens médicaux et pratiques infirmières, moments d'attente ou de détente des équipes de soin), complétée par des entretiens semi-directifs (notamment avec des patients) et une analyse documentaire. Le cadre théorique d'analyse des pratiques dans les différentes recherches emprunte à l'analyse stratégique de Friedberg (1993), sous la forme de ce j'avais appelé un " stratégisme méthodologique " (Demailly, 2005), mais aussi à la théorie des champs de Bourdieu, sans écarter la prise en compte du niveau proprement éthique et politico-éthique de la construction des pratiques.

Il s'agit donc ici d'un retour réflexif et synthétique sur les données issues de cet ensemble de travaux empiriques achevés. L'objet de cet article est de situer la place de l'institution psychiatrie dans les méthodes contemporaines de gouvernement des conduites. On commencera par explorer les injonctions institutionnelles et sociétales auxquelles est soumise la psychiatrie, puis on décrira en deuxième partie les effets structurels de ces injonctions sur les politiques de santé mentale avant d'en venir aux politiques de la relation qui accompagnent les déplacements structurels.

$17^{\mathrm{e}}$ Programme cadre de recherche et développement (PCRD), Coordinateur Bernard Delvaux (Cerisis) http://www.knowandpol.eu/. Elle s'est déroulée de 2005 à 2012.

${ }^{2}$ Pour alléger la lecture, nous ne citerons pas systématiquement ces travaux à l'appui de nos développements dans le cours de l'article. 


\section{Les nouvelles injonctions sociétales à l'égard de la psychiatrie et leur contexte}

Comme toute institution, la psychiatrie est soumise à des attentes sociales quant à la manière dont elle doit remplir ses missions sociétales. S'il s'agit pour elle, depuis le moment historique où ils lui ont été confiés, de traiter les problèmes que pose aux sociétés "l'étrangeté d'âme " (Demailly, 2012). La manière de le faire donne lieu à des injonctions ${ }^{3}$ plurielles, contradictoires et évolutives qui pèsent sur les services psychiatriques et plus largement ceux de santé mentale et tendent à en infléchir les politiques.

Ces injonctions sont conditionnées par des contextes socio-économiques et socio-institutionnels qui permettent d'en comprendre le sens, d'en appréhender les dynamiques dans l'opinion publique et dans la classe politique. Nous allons d'abord donner quelques aperçus sur les éléments de contexte qui semblent pertinents pour la question, avant d'en venir au contenu même des injonctions.

\section{Le contexte socio-économique: l'accroissement des inégalités}

Les aspects du contexte socio-économique dont on peut penser qu'ils infléchissent la situation de la psychiatrie et les attentes sociétales par rapport à elle sont largement décrits par les sociologues, les économistes et les médias : une crise économique et sociale en Europe qui accroît les inégalités, voire la pauvreté, fait monter en de nombreux endroits les taux de chômage ; de nouveaux managements, privés et publics, très axés sur la performance, et d'autant plus durs avec les opérateurs qu'il existe une " armée de réserve " importante; des réticences par rapport à l'immigration qui se crispent d'autant plus que les problèmes d'emploi se développent et qui favorisent la montée du vote d'extrême droite en Europe (Helly, 2014).

Les tensions accrues sur le marché de l'emploi ainsi que le culte de la performance et de la concurrence produisent une suradaptation d'une partie des individus aux normes des milieux de travail, suradaptation qui peut brusquement se fissurer en burn out (Aubert, 2003). Elles favorisent parallèlement, chez ceux qui sont exclus du marché du travail ou qui ne peuvent trouver plaisir à leur activité, la montée des troubles anxieux dépressifs et des addictions (Otero, 2012).

Les logiques inégalitaires de reproduction du système social, de la division en groupes sociaux inégalement favorisés et l'inégalité internationale du partage des ressources entraînent ainsi dans les populations la multiplication des malaises et souffrances. Ceux-ci, médicalisés et nommés par les classifications nosographiques psychiatriques internationales, deviennent des "troubles » et des "syndromes " dont il paraît évident qu'ils doivent être soignés par la psychiatrie. La psychiatrie relève alors de la "santé mentale ", appellation qui entérine plusieurs évolutions conjointes : le caractère extensif des troubles et souffrances psychiques pour lesquels on va faire appel à son magistère, le caractère extensif des professionnels et des dispositifs mobilisés pour traiter les troubles psychiques (qui vont intégrer l'intervention sociale à côté de l'intervention sanitaire) et l'introduction, dans les prises en charge, de préoccupations de santé publique (prévention des maladies, détection précoce, réhabilitation).

De fait, en France comme dans d'autres pays européens, les files actives des services psychiatriques d'adultes s'accroissent (Direction de la recherche, des études, de l'évaluation et des statistiques [DREES], 2011), les pédopsychiatres constatent l'allongement des délais pour les consultations et les médecins généralistes sont de plus en plus souvent confrontés dans leurs cabinets à la souffrance psychique.

\section{Le contexte institutionnel : gouverner les conduites par la santé}

L'accroissement des files actives de la psychiatrie n'est pas qu'un effet mécanique des malaises sociaux, elle est due aussi, comme nous venons de l'esquisser, au fait que ceux-ci s'expriment de plus en plus dans le langage de la santé mentale et sont accueillis dans ce cadre.

\footnotetext{
${ }^{3}$ C'est aussi le cas pour toute institution (Bonny et Demailly, 2012).
} 
Pour penser ce phénomène, il faut faire intervenir deux considérations plus vastes : la prédominance institutionnelle de la santé dans les sociétés développées contemporaines et le rôle accru du travail relationnel pour gouverner les conduites.

\section{La place de la santé}

Sfez écrivait déjà en 1995 que le nouveau salut, c'est la santé. Il attirait l'attention sur le changement de statut symbolique de la santé dans les sociétés de la modernité avancée.

Auparavant, certes, il existait bien une institution de la santé, selon laquelle les médecins étaient chargés d'énoncer le bien des êtres vivants. Cette institution produisait donc une domination spécifique des professionnels sur les profanes. Son mode d'action était particulièrement " disciplinaire ", au sens foucaldien du terme, dans le domaine mental. Cette institution de la santé, pour puissante qu'elle fût, n'était cependant pas prédominante dans la société française, car d'autres, comme l'école, le Droit, l'État ou même les Églises se montraient comparativement plus puissantes, plus prolixes et plus importantes pour la cohésion sociétale. La santé restait en partie une affaire privée, relevant du colloque singulier médecin-malade, la connexion de la santé avec d'autres politiques publiques restant limitée au logement et à l'urbanisme autour de l'hygiène publique (Murard et Zylberman, 1996) et n'envahissait pas l'ensemble de la vie sociale.

Le passage de la santé, que le soin soit public ou privé, au statut d'institution prédominante peut se décrire à la fois dans sa dimension économique (part de plus en plus importante à la fois dans le produit national brut et dans les budgets des ménages), dans sa place dans le débat public et dans sa dimension morale. Au cours des dernières années du $20^{\mathrm{e}}$ siècle, la santé publique connaît une institutionnalisation sans précédent (Fassin et Hauray, 2010). Aux devoirs de la collectivité en matière d'hygiène publique, aux devoirs des individus par rapport à la collectivité (par exemple la vaccination) viennent en effet s'ajouter comme norme sociale les devoirs vis-à-vis de soi-même.

La question de devoirs vis-à-vis de soi est une question débattue par les philosophes. Peut-on fonder en rationalité l'existence de devoirs qui seraient purement vis-à-vis de soi ? Non, répond Ogien (2007), et ils peuvent être ressentis comme un envahissement illégitime de la vie privée. Surveillance et autocontrainte font cependant partie du mode actuel occidental de gouvernement des conduites. Le principe des campagnes de prévention est de modifier les comportements de santé en agissant sur les connaissances, les valeurs et les croyances des individus dans une société, en faisant appel à leur responsabilité. La prévention se présente comme favorable au destinataire. C'est pour son bien, en principe, que l'on veut qu'il change ses comportements et se montre responsable de sa santé. Les campagnes qui portent sur les devoirs vis-à-vis de soi (avoir le souci de soi, de son corps, gérer les risques cardiovasculaires, éviter l'obésité, les accidents domestiques, le tabac, les arrêts de travail, etc.) offrent en même temps des arguments par rapport à la collectivité : "Vous coûtez cher à la société » (ou à la Sécurité sociale).

Cela se fait au prix de l'évitement de l'intervention sur les causes objectives d'une mauvaise santé. Benoist (2012), étudiant la moralisation sanitaire des précaires, affirme que, la plupart du temps, la prise en charge des malades précaires consiste à normaliser des comportements et des individus alors qu'il faudrait simplement normaliser des niveaux de vie et des conditions d'existence.

La norme sanitaire s'étend au domaine de l'esprit. La psychologisation de bon nombre de rapports sociaux permet ainsi de pathologiser les conduites et les affects (l'agitation de l'enfant en TDAH [trouble du déficit de l'attention avec hyperactivité $]^{4}$, la surcharge objective au travail en burn out, l'inégalité d'humeur devant les agressions de l'environnement social en trouble bipolaire), puis la biologie permet de naturaliser les pathologies et de dédouaner l'environnement sociétal d'être source de souffrance psychique. L'alchimie symbolique opérée dans cette double opération est remarquable: la souffrance psychique devient un "problème de santé publique majeur ", un

\footnotetext{
${ }^{4}$ ou en handicap mental léger, comme le montre Eröss (2011) à propos des enfants roms en Hongrie.
} 
problème social donc, mais, dans cette vision du trouble psychique, l'environnement social n'y est pour rien, car sont en cause les gènes ou les molécules de l'accusé ${ }^{5}$ et ses capacités morales à se prendre en charge.

De nouveaux outils viennent étayer cette moralisation sanitaire de la vie quotidienne : le développement de la presse d'information médicale dans les années 1950 (Marchetti, 2010; Ollivier-Yaniv et Rinn, 2009; Saout, Charbonnel et Bertrand, 2008), le développement de technologies relationnelles spécifiques pour l'emprise publique sur les pratiques de soin privées comme l'invention de l'éducation thérapeutique à partir des années 1970 jusqu'à sa promotion comme " politique nationale » en 2008 (Fournier et collab., 2007; Poggi et collab., 2009) et celui de campagnes médiatiques de santé publique. Les premières, en 1950, concernent la sécurité routière et le cancer. Puis viennent, dans les années 1970, des campagnes sur l'alcool au volant, le tabac, le cancer. Dans les années 2000 : tabac, tabagisme passif, sida, vaccinations, risques sportifs, antibiotiques, contraception, drogues, casque en vélo, mieux manger, sécurité routière, cancer. Depuis se sont ajoutés à cette liste: accidents domestiques, grippe aviaire, grossesse et santé, noyade, repères nutritionnels, activité physique, canicule, dépression, tolérance au trouble psychique, accident vasculaire cérébral, protection de l'ouïe. Le nombre annuel de campagnes de santé publique a été ainsi multiplié par 10 depuis les années 1950 (d'après Ketterer, 2012).

Du côté du travail des professionnels, l'extension du paradigme de la santé publique aboutit au rôle majeur de l'épidémiologie et des statistiques dans le raisonnement de santé publique. Parallèlement, il s'agit de formaliser et de standardiser les pratiques (codes de bonnes pratiques, arbres décisionnels, assistance informatique au diagnostic, enregistrement de la totalité des actes soignants) (Bélart, 2011). L'ensemble du traitement informatisé des données de santé par la Sécurité sociale ou les logiciels de gestion hospitaliers permet de publiciser l'état de santé d'une population d'un territoire donné dans le cadre du fort développement de l'épidémiologie psychiatrique, comme le montrent de fait l'ensemble des travaux de la DREES du ministère français de la Santé.

C'est parce que la santé comporte de plus en plus d'enjeux économiques centraux de notre société (Cianferoni, 2013), dans le cadre la primauté accordée aux contraintes d'efficacité et de performance, que l’État et les organisations supra- ou internationales s'y intéressent d'avantage. C'est parce que, dans la rationalité néolibérale, l'État aide à la construction et l'extension des marchés, que le système de santé est de plus en plus régulé par l'État. C'est parce que la santé devient de plus en plus à la fois une affaire publique et un marché et qu'elle concerne de plus en plus la vie personnelle et la subjectivité, incitant chacun à se percevoir comme être détenant un capital-santé et tenu de le faire fructifier, que ce soit avec l'aide de la médecine ou par tout autre moyen utile.

\section{La place du travail relationnel dans le fonctionnement social}

Un deuxième changement institutionnel d'ampleur peut permettre de comprendre les nouvelles injonctions sociétales envers la psychiatrie: c'est l'utilisation croissante du travail relationnel comme mode de gouvernementalité. Les institutions voient décliner leur fonctionnement "disciplinaire » de dressage des corps (Foucault, 2004a et 2004b) pour laisser place à ce qu'il appelle une "biopolitique ", une manière systémique des gérer des populations et des territoires, en calculant des probabilités statistiques et des risques et en maniant des « dispositifs de sécurité ».

Plusieurs observations permettent de formuler l'hypothèse que le travail relationnel est l'opérateur complémentaire de la biopolitique (Demailly, 2008). La croissance de l'emploi dans les métiers relationnels est un argument en faveur de cette hypothèse. Dans un cadre de développement des activités de service de l'économie et dans une société qui se représente le monde de manière prégnante en termes de psychologie, c'est-à-dire de création, de maniement et de réparation des intériorités, c'est aux métiers relationnels que revient le rôle de mettre des pansements ou des prothèses pour contenir les violences et les déchirures sociales potentielles. On pourrait parler de manière significative d'une "société du travail relationnel», tant celui-ci est aujourd'hui imbriqué étroitement, au même titre que la connaissance et l'information, au travail productif : " management » des subjectivités des salariés et des cadres, usage de la psyché comme force productive, exigence du travail en

\footnotetext{
${ }^{5}$ La biologisation univoque du trouble psychique est particulièrement forte en Allemagne : voir à ce propos Nassehi, Demszky et Barth (2011).
} 
équipe et du travail interprofessionnel et interorganisationnel en réseau et en partenariat, exigence du travail sur soi pour gérer au mieux l'entreprise de soi-même. Le travail relationnel s'entrelace aussi avec les problèmes de la société en général, avec la nécessité de maintenir le lien social, susceptible de se dissoudre sous les coups de boutoir des transformations économiques et avec la diminution de la sollicitude envers autrui propre à une société individualiste (Doucet, 2007). Le rôle des activités professionnelles relationnelles n'est pas de type " disciplinaire » ou contraignant, mais plutôt persuasif, incitatif et, comme nous le développerons plus loin, relève plutôt du registre de l'« intervention ».

\section{Les injonctions contradictoires qui traversent le champ de la santé mentale}

C'est dans le contexte socioéconomique et socioinstitutionnel qui vient d'être décrit que peuvent se comprendre les demandes sociales adressées aujourd'hui à la psychiatrie. Nous allons voir qu'elles sont en partie contradictoires.

1) II s'agit d'abord que la psychiatrie serve les exigences d'ajustement des sujets au mode de production et au système social, autrement dit qu'elle contribue à la paix sociale et à promouvoir une autonomie comprise comme adaptation au marché du travail ou aux autres institutions, comme l'école.

La tendance des psychiatres, en réponse à cette injonction et profitant d'elle pour développer leur discipline, sera de contribuer à l'extension du domaine du trouble psychique identifié comme tel. Cette opération est promue par des groupes de psychiatres dans les versions successives du DSM, et aussi dans tous leurs discours publics affirmant par exemple qu'un tiers de la population aura un trouble psychique durant sa vie. Parallèlement, elle est assurée par une diminution de la tolérance collective ou subjective à la bizarrerie comportementale, à l'instabilité d’humeur ou à l'étrangeté culturelle. On peut remarquer ainsi, d'abord aux États-Unis puis dans d'autres pays occidentaux, une explosion du diagnostic de TDAH pour les enfants et de celui de bipolarité chez les adultes.

2) La psychiatrie doit répondre à une autre demande, celle d'ordre et de sécurité, plus en conformité avec sa liaison traditionnelle à l'ordre public, mais dans un nouveau contexte, sécuritariste, alimenté par les peurs sociales vis-àvis des immigrés, des classes dangereuses, des sans-travail. La nouveauté ici est dans la force de l'exigence à ce que soit exercée une contrainte institutionnelle sur les sujets perçus comme déviants ou potentiellement déviants.

3) Une autre injonction, en partie contradictoire avec la précédente, est que la psychiatrie doit s'inscrire dans l'évolution générale des relations humaines dans les sociétés d'État de droit: moins d'asymétrie, moins de domination ou, au minimum, moins d'arrogance, moins de contraintes, plus de respect des professionnels pour les usagers, plus de reconnaissance des savoirs profanes. Ce mouvement est particulièrement net dans le champ de la santé mentale. Les demandes des patients (usagers, destinataires, bénéficiaires, citoyens, de tous milieux sociaux) concernent le droit aux soins, le droit à la continuité du travail relationnel professionnel autour d'eux, le droit à l'information, le droit à l'articulation du projet de soin et du projet de vie, un déclin de l'asymétrie de leurs relations avec les psychiatres et les infirmiers (Rabeharisoa 2010), le droit au respect et à la co-construction du soin.

4) Ajoutons à ces trois premières injonctions celle d'une baisse forte des coûts publics de la santé, sensible dans tous les pays européens, mis à part peut-être en Norvège.

Cette dernière injonction n'est pas forcément compatible avec le déploiement des dispositifs impliqué par la première injonction (la pathologisation de la vie humaine ordinaire), ni avec l'augmentation de la qualité et de l'humanité des soins suggérée par la troisième. Quant à la contradiction entre la deuxième et la troisième, elle semble claire.

\section{Déplacements institutionnels}

En réponse à ces injonctions nouvelles et contradictoires, plusieurs catégories d'acteurs redéfinissent leurs stratégies, leurs priorités et leurs positionnements. Cela aboutit à des déplacements et des reconfigurations au sein du champ de la santé mentale, qui produisent en retour des redéfinitions des " mandats » (Hughes, 1997) de 
plusieurs catégories de professionnels en une sorte de «tectonique des plaques » concernant la prise en charge de "l'étrangeté d'âme ".

Vers la fin de la "folie " et de la « maladie mentale "?

Après plusieurs remaniements, qui ont différemment découpé le périmètre de "l'étrangeté d'âme " et lui ont attribué des types différents de professionnels, l'âge de la psychiatrie classique est devenu celui où les sujets troublés, sous l'appelation de "malades mentaux ", devenaient les clients naturels des services de psychiatrie publique, en hôpital ou en ambulatoire, et vice versa. Cette configuration semble révolue.

Les dernières versions des classifications nosographiques entérinent un effritement de la distinction entre la normalité et la «maladie mentale ». Les coupures symboliques raison/déraison (ou folie) et normalité/maladie, laissent place à un continuum évaluable et mesurable du désordre psychique, avec pour conséquence en France une répartition administrativo-gestionnaire des sujets selon la gravité de leurs malaises et leur capacité différentielle de résilience sociale. Les sujets psychiquement troublés ne sont plus principalement, au sein du vaste champ de la santé mentale, suivis par les services de psychiatrie publique, et même si ceux-ci voient globalement leur patientèle augmenter en nombre absolu.

Un premier ensemble continue à relever de leur obédience, mais ce ne sont plus exactement les mêmes patients qu'avant, la proportion de ceux présentant des troubles anxieux et dépressifs étant beaucoup plus importante. Ils sont pris en charge au sein de cursus dans lesquels le temps d'hospitalisation est réduit, par exemple à la période de crise, pour d'une part économiser surles budgets publics et d'autre part travailler l'autonomie, la réadaptation et le rétablissement dans le cadre de dispositifs ambulatoires, suivant en cela des tendances internationales (Rodriguez del Barrio et Poirel, 2011). Les services psychiatriques gardent un deuxième sous-groupe de destinataires, ceux qui relèvent de l'hospitalisation sous contrainte, laquelle, en France, est ces dernières années en augmentation régulière ( $\mathrm{du}$ moins sous sa forme "hospitalisation d'office " ${ }^{6}$ ), ce qui répond à l'idéologie sécuritariste.

Un deuxième ensemble de sujets troublés, plus important que le précédent d'après l'enquête administrative Handicaps-Incapacités-Dépendances, est pris en charge en établissement médico-social, avec des taux élevés de psychoses et de dépressions (sans parler des troubles neurologiques ou du handicap mental): foyers d'hébergement, maisons de retraites, établissements pour handicapés mentaux et psychiques, qui recueillent une grande partie de la population qui autrefois aurait séjourné de façon permanente à l’hôpital psychiatrique, mais en est maintenant écartée.

Un troisième ensemble, lui aussi important, de sujets troublés est en prison (10\% des détenus souffriraient de schizophrénie, 1 sur 4 pourrait être diagnostiqué comme psychotique, 1 sur 2 comme affecté de troubles anxieux ou dépressifs, 1 sur 3 souffrirait de toxicomanie). La demande sociale par rapport aux malades psychiques, à partir du moment où ils sont délinquants ou criminels, est d'abord qu'ils soient punis et enfermés (ou chassés et bannis s'ils sont migrants) avant d'être soignés. C'est un sujet qui divise les psychiatres, certains restant attachés à l'irresponsabilité pénale des malades mentaux, d'autres défendant la priorité de la sanction pénale, soit pour protéger et satisfaire les victimes, soit au nom de l'exemplarité de la punition, soit au nom de la citoyenneté du malade mental. Quoi qu'il en soit, l'enfermement pénitentiaire est un mode important de gestion des sujets troublés.

Un quatrième ensemble de sujets troublés vit dans le monde " ordinaire ", n'a pas accès aux soins médicaux, ne relève que du seul travail social ou est même carrément abandonné. Bon nombre de sujets en souffrance parmi les travailleurs ou les chômeurs migrants pauvres, parmi les adolescents de milieu populaire ou parmi les toxicomanes appartiennent à cette catégorie, qui tombe dans les « trous » de la prise en charge sanitaire, voire même sociale.

\footnotetext{
${ }^{6}$ Voir Delarue (2012) et Barbier, Demontes, Lecerf et Michel (2010). En France, il y a 11 hospitalisations sans consentement pour 100000 habitants, ce qui représente $12,5 \%$ des hospitalisations psychiatriques. II existe, depuis 1992, une tendance à l'augmentation régulière du nombre de mesures d'hospitalisation d'office : de plus de $8 \%$ entre 2003 et 2007 en moyenne à l'échelle nationale, mais une multiplication par quatre à Paris (Commission des Citoyens pour les Droits de l'Homme, 2009).
} 
Enfin, un dernier groupe est constitué de ceux qui parviennent à la fois à esquiver la psychiatrie, le médico-social et le travail social et qui s'adressent, pour obtenir un soulagement de leurs troubles, aux psychothérapeutes de toutes obédiences, aux psychanalystes, aux thérapies corporelles et aux médecines alternatives.

Le champ se divise donc à la fois selon la solvabilité des groupes de destinataires et selon leur aptitude à l'autonomie, définie comme adaptation sociale et absence de déviance.

Pour ajuster les zones de transition et mieux gérer l'orientation des clientèles entre les différents modes de prise en charge, il devient utile de développer l'ambulatoire sous forme d'équipes mobiles (Demailly, Dembinski et Le Roux, 2014), de créer des centres d'urgence et de crise et de favoriser un travail social de type occupationnel ou rééducatif.

\section{Des services différenciés selon les groupes sociaux}

On vient de mentionner la solvabilité comme facteur d'orientation entre les différents dispositifs privés ou publics de traitement de la maladie, traitement au sens social du terme et non au sens thérapeutique bien sûr. Un psychotique pauvre ou immigré a plus de chance de se retrouver en prison que d'être suivi au centre médicopsychologique d'un secteur psychiatrique (Aïach, 2010).

Par ailleurs, la solvabilité et la diversité socioculturelle agissent sur le type de psychothérapie auxquelles les personnes peuvent avoir accès. Les techniques cognitivo-comportementales (TCC) ${ }^{7}$ concernent de fait surtout les membres des classes populaires ou les migrants qui ont accès aux soins et qui, en cas de souffrance psychique et de demande de soins, fréquentent les établissements publics de santé mentale (accès gratuit), lesquels s'orientent massivement vers les TCC, pour des raisons de coût ${ }^{8}$ et parce qu'il faut normaliser rapidement les comportements. Les TCC sont destinées également aux exclus et aux personnes âgées, que les institutions du médico-social tentent d'amener à ce type de soin.

Mais la mouvance cognitivo-comportementale concerne aussi les membres des classes moyennes et moyennes supérieures désireux de se maintenir à flot dans la rude concurrence sur le marché du travail. C'est le cas du coach (en libéral) ou des cliniques privées à but lucratif pour les troubles anxieux dépressifs ou pour le burn-out.

Seuls les groupes sociaux disposant d'un certain capital culturel et de ressources suffisantes parviennent en France à se dispenser des TCC et à se tourner vers des psychothérapies humanistes et relationnelles (psychanalyse, Gestalt, thérapie de couple, etc.) ou vers diverses thérapies corporelles et techniques du soi dans lesquelles l'autonomie n'est pas interprétée principalement selon le registre de l'adaptation et de la soumission aux normes, mais plutôt comme forme de connaissance de soi et comme émancipation.

Mais ce n'est pas là qu'une question sociologique, c'est aussi une question éthique.

\section{Politiques du relationnel et questions éthiques}

\section{Le travail relationnel et la montée du registre de l'“ intervention »}

L'extension quantitative des métiers de relation à autrui s'accompagne d'une tension sur les registres de la relation mise en jeu, particulièrement dans le champ de la santé mentale. J'ai déjà distingué (Demailly, 2008) de manière idéal-typique douze registres du travail de relation (éducatif, didactique, thérapeutique, analytique, mercaticien, assistantiel, de pilotage, de persuasion, hiérarchique-bureaucratique, d'accompagnement-soutien, sécuritaire, de l'intervention), en fonction d'une part du sens de l'action et de la source de sa légitimité et d'autre part du statut symbolique de l'autrui concerné.

\footnotetext{
${ }^{7}$ Pour un panorama des TCC, voir Cottraux (2001).

8 Les établissements d'orientation clairement psychodynamique, phénoménologique ou systémiques deviennent rares, les consultations d'ethnopsychiatrie le sont également
} 
Globalement, le registre de l'intervention (ou de l'ingérence), selon la définition qui en avait été donnée et qui est un peu différente de l'usage ordinaire du terme, tend à en évincer d'autres. Il est proche de celui de la persuasion, mais il implique l'idée d'action immédiate dans le réel matériel et pas seulement d'action symbolique sur les mots et les images. Il se différence du registre assistantiel-charitable : ce n'est pas la même chose de laisser tranquille une personne sans abri qui ne veut pas aller dans un foyer d'hébergement (registre assistantiel) ou de l'y emmener contre son gré, pour son bien, au nom d'intérêts supérieurs qui justifient l'ingérence. Ce registre se différencie aussi des pratiques qu'on l'on peut classer dans l'accompagnement ${ }^{9}$, dans lequel le respect de la demande est central, alors que l'intervention prétend connaître les besoins de la personne, parfois mieux que cette dernière. Le déclin du registre assistantiel se remarque par le fait qu'il devient de moins en moins légitime. Même si des initiatives comme les Restos du Coeur, les Compagnons d’Emmaüs ou l'Armée du Salut restent largement visibles, on note en effet au fil des trente dernières années la multiplication des " dispositifs " qui fonctionnent plutôt sous une forme contractuelle avec leurs bénéficiaires, et formulent donc des exigences : on n'aide pas " pour rien », on ne fait pas de "l'assistanat", on aide les "bons" usagers ou les "bons " migrants compliants. En ce sens, l'intervention est bien en lien avec deux tendances sociétales puissantes dans les sociétés occidentales : le devoir d'agir (d'être à l'initiative, d'être responsable, la valorisation du "faire " plutôt que du non-agir) et le droit ou devoir d'ingérence, tendances qui définissent les politiques de la relation.

La multiplication des dispositifs sécuritaires (police de proximité, vigiles, agents d'ambiance, agents de médiation dans les métros) s'inscrit bien sûr aussi dans ce panorama de l'extension du registre de l' " intervention » et de sa mise en visibilité. L'obligation d'un management proactif allant chercher la mobilisation des salariés et l'extension du marketing ostensible de la consommation dans la vie quotidienne et dans la vie publique constituent également des phénomènes convergents quant à la légitimité acquise de l'ingérence comme mode de relation. L'obligation de travail interinstitutionnel et en réseau favorise une solution de facilité pour faciliter les coopérations interprofessionnelles: c'est que tous les "intervenants» autour d'une personne passent au registre de l'« intervention », sorte de sécuritaire soft où les professionnels ne prennent pas de risque.

Quel sens donner à cette tendance qui affecte le travail dans le champ de la santé mentale? II est assez logique de le rapprocher du concept de "gouvernementalité » de Foucault et de voir dans l'intervention un dispositif de gouvernementalité. J'en rappelle la définition :

Par gouvernementalité, j'entends l'ensemble constitué par les institutions, les procédures, analyses et réflexions, les calculs et les tactiques qui permettent d'exercer cette forme bien spécifique, bien que complexe, de pouvoir qui a pour cible principale la population, pour forme majeure de savoir, l'économie politique, pour instrument technique essentiel les dispositifs de sécurité. (Foucault, 1994, p. 655)

Il y a eu deux grandes révolutions dans la technologie du pouvoir: la découverte de la discipline et la découverte de la régulation, le perfectionnement d'une anatomo-politique, et le perfectionnement d'une bio-politique. (Foucault, 2001, p. 193)

Plutôt que du côté des "disciplines ", comme action directe sur les corps, l'intervention se décline du côté de la régulation. L'importance des affaires de l'âme, des technologies du soi et des technologies de l'action sur autrui relève d'une épistémè à la fois libérale et autoritaire. Le premier ressort de son développement est la prise en compte des limites des ordonnances sociales directes des "disciplines " face aux exigences modernes de l'individualisation et de la singularisation (Martucelli, 2010) et face à la pluralisation des processus de socialisation. Le second ressort est de tenir compte des limites des "biopouvoirs ", confrontés à l'indomptabilité de la nature et l'imprévisibilité des êtres humains. La dimension praxique de l'intervention est donc, de fait, la modalité pratique et politique de réalisation de l'action sociale en modernité avancée.

\footnotetext{
${ }^{9}$ Les mots employés par les professionnels sont aussi ceux de " soutien », de " care ». Leur usage est le plus souvent ambigu et peut correspondre à plusieurs des catégories analytiques que j'ai distinguées analytiquement quant aux registres du travail relationnel.
} 


\section{Les résistances au registre de l'intervention}

Mais il ne s'agit nullement d'un rouleau compresseur. L'intervention comme ingérence pour le bien d'autrui n'est pas sans susciter quelque résistance, notamment des associations des usagers de la psychiatrie. Un mot d'ordre fait actuellement fortune en France après son invention outre Atlantique "Rien sur nous sans nous!", qui est une critique directe du registre de l' « intervention ", au sens où les professionnels règleraient entre eux le sort des sujets, en principe pour le bien de ceux-ci, sans les consulter. Les patients, individuellement parlant, et les associations d'usagers expriment de façon de plus en plus explicite, voire virulente, les exigences de respect, de reconnaissance et de participation (Greacen et Jouet, 2012). Ils appellent à d'autres politiques du relationnel.

De ce fait, ces dernières années, par exemple depuis la loi de 2005 en France sur les droits des «usagers», le registre de l'intervention s'assouplit, s'euphémise, devient discret dans la quotidienneté des pratiques. De plus, et de manière moins rhétorique, plus réellement subversive, des formes de rapports horizontaux entre professionnels et profanes sont expérimentées (c'est le cas dans l'opération des médiateurs de santé pairs en santé mentale), elles suscitent des controverses, mais, malgré les oppositions d'une partie des professionnels, réussissent à démontrer leur faisabilité, leur intérêt, leur valeur éthique (Demailly et collab., 2014). La modification de la place des usagers est plus effective en Écosse, en Norvège et au Québec et fournit des modèles aux innovateurs français.

\section{Les questions éthiques et les conceptions de l'autonomie}

Le champ de la santé mentale est donc aujourd'hui traversé par une série de questions éthiques, formulées par des psychiatres, des philosophes et des associations d'usagers, et que le sociologue pourrait reformuler ainsi : quel est le sens du travail professionnel auprès des sujets atteints de troubles psychiques : sur eux, contre eux, malgré eux, sans eux, pour eux, avec eux? Que faire par rapport à leurs moments de révolte? Est-ce que le but de l'intervention sanitaire et sociale sur un sujet est qu'il se résigne à sa condition, que son comportement ne soit pas source de trop de désordre dans la société ou bien qu'il prenne sa vie en main?

Les questions pratiques que rencontrent les professionnels dans la quotidienneté de leur travail se rattachent à leur conception de l'autonomie. Est-elle un mouvement de simple adaptation ou d'émancipation? On pourrait, de manière idéale-typique, distinguer deux visions de l'autonomie, deux politiques relationnelles, qui tendent à partager les soignants en deux catégories. Ces deux éthiques de l'autonomie ne conduisent pas aux mêmes modalités d'interprétation de la diversité culturelle et sociale, que ce soit dans son rapport à la singularité ou dans son rapport au vivre ensemble.

- D’un côté, la notion d'autonomie résonne avec la liberté de pensée, une forme d'individualisme libertaire, le droit à la parole et à être écouté, l'affirmation de l'ontologie de la singularité et de la valeur de la singularité, l'espoir de l'authenticité, l'émancipation des dominés (dont les «malades mentaux»), l'empowerment comme revendication et construction.

- De l'autre, la sécurisation de la société et des psychismes, le souci de surveillance du corps et de ses performances, l'hygiène mentale, l'empowerment comme octroi ${ }^{\mathbf{1 0}}$, l'affirmation de la valeur de la normalisation de la standardisation, de la visibilité, de l'adaptation à une communauté, les bienfaits d'une relation contractuelle au thérapeute avec des épreuves précises auxquelles le sujet doit satisfaire pour que son expérience soit validée.

\section{Conclusion}

Les pratiques et politiques de santé mentale sont aujourd'hui traversées de contradictions fortes, d'autant plus que la santé est devenue une institution prédominante sur laquelle convergent des injonctions sociétales variées. Leur juxtaposition et leurs contradictions ont abouti à un redécoupage des clientèles et patientèles entre institutions, qui se déploient de manière complémentaire et se partagent l'intervention, plus ou moins autoritaire ou

\footnotetext{
${ }^{10}$ Pour la Commission Européenne (2001), l'autonomisation, en anglais empowerment, est « la démarche qui consiste à octroyer aux individus le pouvoir de prendre des initiatives responsables en vue d'orienter leur vie et celle de leur communauté (ou société) dans les domaines économiques, social et politique » (c'est moi qui souligne) (cité par Greacen et Jouet, 2012p. 254).
} 
participative, sur les sujets troublés, le gardiennage, l'enfermement, la punition, la réadaptation et parfois, de plus en plus rarement semble-t-il, les soins. Des conceptions éthiques s'opposent autour de l'interprétation d'un des maîtres mots de la modernité, l'autonomie, conçue comme adaptation ou comme émancipation. Le système d'imposition culturelle n'est plus seulement polyphonique, comme les sociologues et les historiens le savent depuis longtemps, mais il est dysharmonique, et c'est peut-être cela la caractéristique principale de la modernité avancée.

\section{Bibliographie}

Aïach, P. (2010). Les inégalités sociales de santé. Paris : Economica.

Aubert, N. (2003). Le Culte de l’urgence : La société malade du temps. Paris : Flammarion.

Autès, M. et Demailly L. (dir.). (2005). La construction sociale du trouble psychique. IFRESI/Contrat de plan État Région Nord-Pas-de-Calais.

Autès, M., Demailly L., Dembinski O., Bélart C. et Lardé, P. (2010). Les outils de régulation de l'action publique en santé mentale en France : Outils gestionnaires et outils statistiques, Rapport D13 KnowandPol, CLERSE/CE ${ }^{\mathrm{e}}$ PCRD. http://www.knowandpol.eu/

Barbier, M., Demontes, C., Lecerf M. et Michel, J.-P. (2010). Rapport d'information fait par le groupe de travail sur la prise en charge des personnes atteintes de troubles mentaux ayant commis des infractions. Rapport $n^{\circ} 34$ présenté au Sénat le 5 mai 2010.

Bélart, C. (2011). La psychiatrie à l'épreuve des outils de gestion. Dans S. Maugeri, J.-L. Metzger et M. BenedettoMeyer (dir.), L'emprise de la gestion. La société au risque des violences gestionnaires (p. 215-244). Paris : L'Harmattan.

Benoist, Y. (2012). Précarité, médecine et normalisation. Communication présentée aux Journées d'étude du RT 19 de l’Association française de sociologie « La santé, affaire privée, affaire publique? », Paris, 23 octobre .

Bonny, Y. et Demailly, L. (dir.). (2012). L'institution plurielle. Lille : Presses du Septentrion.

Bouvier, G. (2011). L'enquête Handicap-Santé : présentation générale. Paris : INSEE.

Cianferoni, N. (2013). La santé, nouvelle variable d'ajustement dans la course à la compétitivité. Dans J. Ferrette (dir.), La place des rapports hiérarchiques dans la souffrance au travail. L'exemple du secteur public (p. 101111). Paris : L'Harmattan.

Commission des Citoyens pour les Droits de l'Homme. (2009). Les internements psychiatriques en France, rapport de synthèse. Paris.

Commission européenne. (2001). Communication de la Commission - Réaliser un espace européen de l'éducation et de formation tout au long de la vie [en ligne].

http://europa.eu/legislation_summaries/education_training_youth/lifelong_learning/c11054_fr.htm

Cottraux, J. (2001). Les thérapies cognitives (Comment agir sur nos pensées?). Paris : Retz.

Delarue, J.-M. (2012). Rapport d'activité 2012. Paris : Dalloz.

Demailly, L. (2005a). Cloisonnements et coopérations dans le champ de la prise en charge des troubles psychiques, L'Information psychiatrique, 81(4), 337-343.

Demailly, L. (2005b). Pour une représentation politique de l'acteur au travail, CLES, 45, 153-174.

Demailly, L. (2008). Politiques de la relation. Sociologie des métiers et du travail de relation. Lille : Presses Universitaires du Septentrion.

Demailly, L. (2011). Sociologie des troubles mentaux. Paris : La Découverte.

Demailly L. (2012). Regulatory mechanisms, policies and knowledge in the Education and the Mental Health sectors. Dans J. Barroso et L. Carvalho (dir.), Knowledge and regulatory processes in Health and education policies (p. 131-147). Lisbonne : Educa.

Alterstice - Revue Internationale de la Recherche Interculturelle, vol. 4, $n^{\circ} 2$ 
Demailly, L. (2014). Exigence de travail sur soi, santé mentale et rapports sociaux dans la société française. Dans M.-C. Doucet et N. Moreau (dir.), Sante mentale et société (p. 229-249). Montréal : Presses de l'Université du Québec.

Demailly, L. et Bresson, M. (dir.). (2005). Les modes de coordination entre professionnels dans le champ de la prise en charge des troubles psychiques. Convention IFRESI/ DREES MIRE /INSERM.

Demailly, L., Bélart, C., Déchamp Le Roux, C., Dembinski, O., Farnarier, C., Garnoussi, N. et Soulé, J. (2014). Le dispositif des médiateurs de santé pairs en santé mentale : une innovation controversée. Rapport final de la recherche évaluative qualitative sur le programme expérimental 2012-2014. Lille : Convention de recherche CLERSE USTL Lille 1/CCOMS EPSM Lille-Métropole.

Demailly, L. et Broise, P. (de la). (2009). Les enjeux de la déprofessionnalisation, Socio-logos, 4 (en ligne).

Demailly, L. et Chéronnet, H. (2007). Politique de proximité et tentative de déstigmatisation dans un secteur de pédopsychiatrie Contrat IFRESI/ EPSM Lille Métropole. Lieu : éditeur

Demailly, L., Chéronnet, H. et Mossé, P. (2009). La territorialisation de la politique de santé mentale. KnowandPol 6ème PCRD Bruxelles/CLERSE/LEST. http://www.knowandpol.eu/

Demailly, L., Dembinski, O. et Déchamp Le Roux, C. (2014). Les équipes mobiles de psychiatrie et le travail de disponibilité. Montrouge : John Libbey.

Demailly, L., Devineau, J., Maury C. et Mossé P. (2010). Accountability and Mental Health in France, Rapport D18 KnowandPol, LEST/CLERSE/CE $6^{\mathrm{e}}$ PCRD.

Doucet, M.-C. (2007). Solitude et sociétés contemporaines, une sociologie de l'individu et du rapport à l'autre. Québec : Presses de l'Université du Québec.

Direction de la recherche, des études, de l'évaluation et des statistiques (DRESS). (2011). L'état de santé de la population en France, Rapport 2011. Paris : DREES.

Eröss, G. (2011). Construction et déconstruction politiques du handicap mental léger chez l'enfant (Hongrie, 20022009), Sociologie Santé, 34, 69-86. (Les politiques de psychiatrie et santé mentale : incertitudes et perspectives)

Fassin, D. et Hauray, B. (2010). Santé publique, l'état des savoirs. Paris : La Découverte.

Foucault, M. (2001). Les mailles du pouvoir. Dans : Dits et écrits II, 1976-1988. Paris: Gallimard.

Foucault, M. (2004a). Naissance de la biopolitique. Cours au Collège de France 1978-1979. Paris : Gallimard/Seuil.

Foucault, M. (2004b). Sécurité, territoire, population. Cours au Collège de France 1977-1978. Paris : Gallimard/Seuil.

Fournier, C., Attali, C., Bocquet-Chabert, A. et collab. (2007). Besoins d'information et d'éducation des personnes diabétiques, pratiques éducatives des médecins, étude Entred, France, BEH : Bulletin épidémiologique hebdomadaire, 42-43, 460-464.

Friedberg, E. (1993). Le Pouvoir et la Règle. Dynamiques de l'action organisée. Paris : Seuil.

Greacen, T. et Jouet, E. (2012). Pour des usagers de la psychiatrie acteurs de leur propre vie. Paris : Érès.

Helly, D. (2014). Les murs de la mobilité. Communication présentée au congrès international de l'ACSALF, Ottawa, 14-17 octobre.

Hughes, E. (1997). Le regard sociologique. Paris : Éditions de l’EHESS.

Jobert, B. (1995). Rhétorique politique, controverses scientifiques et construction des normes institutionnelles. Dans A. Faure, G. Pollet et P. Warin (dir.), La construction du sens dans les politiques publiques (p. 13-125). Paris : L'Harmattan.

Ketterer, F. (2012). Le processus de production publicitaire dans les campagnes médiatiques de santé publique. Entre inspiration socio-politique et expiration médiatique (thèse de doctorat). Université de Lille 1, Lille.

Alterstice - Revue Internationale de la Recherche Interculturelle, vol. 4, $n^{\circ} 2$ 
Le Gales, P. et Lascoumes, P. (dir.). (2005). Gouverner par les instruments. Paris : Presses de Sciences-Po.

Marchetti, D. (2010). Quand la santé devient médiatique. Les logiques de production de l'information dans la presse. Grenoble : Presses Universitaires de Grenoble.

Martuccelli, D. (2010). La société singulariste. Paris : Armand Colin.

Muller, P. et collab. (2005). L'analyse politique de l'action publique. Confrontation des approches, des concepts et des méthodes, Revue française de science politique, 1, 5-6.

Murard, L. et Zylberman, P. (1996). L'hygiène dans la République. La santé publique en France, ou l'utopie contrariée (1870-1918). Paris : Fayard.

Nassehi, A., Demszky, A. et Barth, N. (2011). La politique de santé mentale allemande : passé et présent. Sociologie Santé, 34, 161-180.

Ogien, R. (2007). L'éthique aujourd'hui. Paris : Gallimard.

Ollivier-Yaniv, C. et Rinn, M. (dir.). (2009). Communication de l'État et gouvernement du social. Pour une société parfaite? Grenoble : Presses de l'Université de Grenoble.

Otero, M. (2012). L’Ombre portée : l'individualité à l'épreuve de la dépression. Montréal : Boréal.

Palier, B. et Surel, Y. (2005). Les «trois I» et l'analyse de l’État en action, Revue française de science politique, 1(55), 7-32.

Poggi, M.-P. et collab. (2009). Éducation à la santé en EPS et attentes familiales : confusion entre sphère privée et espace public, Carrefours de l'éducation, 2009/1(27), 153-158.

Rabeharisoa, V. (2010). Les associations de malades et d'usagers de la santé. Dans D. Fassin et B. Hauray (dir.), Santé publique. L'état des savoirs (p. 457-468). Paris : INSERM-La Découverte.

Robillard, D. (2013). Rapport d'information en conclusion des travaux de la mission sur la santé mentale et l'avenir de la psychiatrie, enregistré à la Présidence de l’Assemblée nationale le 18 décembre 2013.

Rodriguez del Barrio, L. et Poirel, M.-L. (2011). Le traitement dans la communauté. Santé mentale au Québec, 36(1), 153-168.

Saout, C., Charbonnel, B. et Bertrand, D. (2008). Pour une politique nationale d'éducation thérapeutique du patient. Rapport présenté à Roselyne Bachelot-Narquin, Ministre de la Santé [France].

Sfez, L. (1995). La santé parfaite. Paris : Seuil. 